S. TANNO

KODAI MATH. J.

13 (1990), 204-209

\title{
INTEGRALS OF SOME TRIGONOMETRIC FUNCTIONS
}

\author{
By Shukichi TANNo
}

\section{Introduction}

Let $a$ and $b$ be positive numbers, and $u, v$ and $m$ be positive integers such that $u+v=m, 2 \leq m$. We define $I(m)$ and $I(u ; v)$ by

$$
I(m)=\int_{0}^{\infty} \frac{\sin ^{m} a t}{t^{m}} d t, \quad I(u ; v)=\int_{0}^{\infty} \frac{\sin ^{u} a t \sin ^{v} b t}{t^{m}} d t .
$$

Tables of integrals give values of $I(m)$ and $I(u ; v)$ only for some special cases. For example, for $3 \leq m \leq 6$, Gradshteyn and Ryzhik [4] (p. 449-p. 450) gives

$$
\begin{array}{ll}
I(3)=3 a^{2} \pi / 8, & I(4)=a^{3} \pi / 3, \\
I(5)=115 a^{4} \pi / 384, & I(6)=11 a^{5} \pi / 40 .
\end{array}
$$

As for $I(u ; v)$ with $a<b$, [4] (p. 451-p. 452) gives

$$
\begin{aligned}
I(2 ; 2) & =(3 b-a) a^{2} \pi / 6 & & (a<b), \\
I(3 ; 1) & =a^{3} \pi / 2 & & (0<3 a \leq b) \\
& =\left[24 a^{3}-(3 a-b)^{3}\right] \pi / 48 & & (0<a<b \leq 3 a), \\
I(1 ; 3) & =\left(9 b^{2}-a^{2}\right) a \pi / 24 & & (a<b) .
\end{aligned}
$$

In this note we give the general expressions of $I(m)$ and $I(u ; v)$. These are special cases of Theorem A below. To state our Theorem A we need the following definition. Let $a_{1}, a_{2}, \cdots, a_{m}$ be positive numbers such that $0<a_{1} \leq a_{2} \leq \cdots \leq$ $a_{m-1} \leq a_{m}$. For a subset $\lambda=\left\{k_{1}, k_{2}, \cdots, k_{m-r}\right\}$ of $\{1,2, \cdots, m-1\}$, a polynomial

$$
P_{r}(\lambda)=a_{k_{1}}+a_{k_{2}}+\cdots+a_{k_{m-r}}-a_{k_{m-r+1}}-\cdots-a_{k_{m-1}}-a_{m}
$$

is said to be of $r$-type, if $\left\{a_{k_{1}}, a_{k_{2}}, \cdots, a_{k_{m-1}}, a_{m}\right\}=\left\{a_{1}, a_{2}, \cdots, a_{m}\right\}$ as sets and

$$
P_{r}(\lambda)>0, \quad k_{1}<k_{2}<\cdots<k_{m-r}, \quad k_{m-r+1}<\cdots<k_{m-1} .
$$

Note that $a_{m}$ appears with negative sign and $r$ is the number of negative signs contained in a polynomial of $r$-type. A polynomial of 1-type is unique if it exists.

Received November 28, 1989. 
THEOREM A. For constants $1<a_{1} \leq a_{2} \leq \cdots \leq a_{m-1} \leq a_{m}, 2 \leq m$, the following holds:

$$
\frac{2}{\pi} \int_{0}^{\infty} \frac{1}{t^{m}}\left(\prod_{k=1}^{m} \sin a_{k} t\right) d t=\prod_{k=1}^{m-1} a_{k}-\frac{1}{c^{\prime}} \sum_{r=1}^{m-2}(-1)^{r-1} \sum_{r-t y p e} P_{r}(\lambda)^{m-1},
$$

where $c^{\prime}=(m-1) ! 2^{m-2}$ and $P_{r}(\lambda)$ denotes a polynomial of $r$-type.

If $a_{m}>a_{1}+a_{2}+\cdots+a_{m-1}$, then there is no polynomial of $r$-type $(r \geq 1)$, and so the above integral does not depend on $a_{m}$.

For proof of Theorem A we use the volume expression of cube-slicing by Hensley [5] and Ball [1], and actually we give the volume of cube-slicing in terms of numbers $a_{\imath}$ (after normalization) using polynomials of $r$-type by elementary geometric method.

A special case of Theorem A for $m=3$ is given, for example, at p. 79 of Erdélyi [3] and at p. 422 of Gradshteyn and Ryzhik [4]. A special case where there is no polynomial of $r$-type $(r \geq 1)$ is given at p. 417 of [4].

Three special cases of Theorem $\mathrm{A}$ are given as Corollaries C, D and $\mathrm{E}$ in $\S 3$. From these one can deduce many analogous formulas. Only several examples are given in Corollary $\mathrm{F}$.

The author is grateful to Professor N. Kurokawa, Professor S. Saitoh and Professor N. Suita for their kind informations.

\section{The volume of cube-slicing}

By $\left\{e_{\jmath}, 1 \leq j \leq m\right\}$ we denote the standard base of the Euclidean $m$-space $E^{m}$ at the origen $O$, and by $\left\{x^{j}\right\}$ the standard coordinate system of $E^{m}$. Let $K^{m}$ be the unit cube in $E^{m}$, which is expressed by $\left\{x ; 0 \leq x^{3} \leq 1,1 \leq j \leq m\right\}$. Let $b_{1}, b_{2}$, $\cdots, b_{m}$ be positive numbers such that

$$
b_{1} \geq b_{2} \geq \cdots \geq b_{m}>0 .
$$

Let $T$ be an $m$-simplex determined by $\left\{O, p_{1}, p_{2}, \cdots, p_{m}\right\}$, where $p_{j}$ is the end point of the vector $b_{\jmath} e_{\jmath}, 1 \leq \jmath \leq m$.

LEMMA. The volume $V\left(K^{m} \cap T\right)$ of the intersection of $K^{m}$ and $T$ is given by

$$
V\left(K^{m} \cap T\right)=\frac{b_{1} b_{2} \cdots b_{m}}{m !} \sum_{r=0}^{m}(-1)^{r}{ }_{k_{1}<k_{2}<\cdots<k_{r}} \sum^{*}\left(1-\frac{1}{b_{k_{1}}}-\cdots-\frac{1}{b_{k_{r}}}\right)^{m},
$$

where $\Sigma^{*}$ denotes the sum over all positive terms $\left(1-1 / b_{k_{1}}-\cdots-1 / b_{k_{r}}>0\right)$.

Proof. (i ) If $b_{1} \leq 1$, then the $m$-simplex $T$ is contained in $K^{m}$ and $V\left(K^{m} \cap T\right)$ $=V(T)=b_{1} b_{2} \cdots b_{m} / m !$.

(ii) If $b_{1}>1 \geq b_{2}$, then only one vertex $p_{1}$ of $T$ lies outside $K^{m}$. The intersection $T \cap\left(K^{m}\right)^{C}$ of $T$ and the complement $\left(K^{m}\right)^{C}$ of $K^{m}$ in $E^{m}$ defines an $m$ - 
simplex $T_{1}$, which we call the outer simplex at $p_{1} . \quad V\left(K^{m} \cap T\right)$ is given by the difference of the volume $V(T)$ and the volume $V\left(T_{1}\right)$ of the outer simplex at $p_{1}$, and

$$
V\left(K^{m} \cap T\right)=\left(b_{1} b_{2} \cdots b_{m} / m !\right)\left[1-\left(1-1 / b_{1}\right)^{m}\right] .
$$

(iii) If $b_{s}>1 \geq b_{s+1}$, for $2 \leq s \leq m$ (putting $b_{m+1}=0$ ), then vertices $p_{1}, p_{2}, \cdots, p_{s}$ of $T$ lie outside $K^{m}$. In this case the outer simplex $T_{h}$ at $p_{h}, 1 \leq h \leq s$, is defined by $T_{h}=T \cap\left\{x ; x^{h} \geq 1\right\}$.

(iii-1) If the outer simplexes at $p_{1}, p_{2}, \cdots, p_{s}$ are disjoint, then $V\left(K^{m} \cap T\right)=$ $V(T)-V\left(T_{1}\right)-V\left(T_{2}\right)-\cdots-V\left(T_{s}\right)$, where $V\left(T_{h}\right)=\left(b_{1} b_{2} \cdots b_{m} / m !\right)\left(1-1 / b_{h}\right)^{m}, 1 \leq h \leq s$.

(iii-2) Two outer simplexes at $p_{1}$ and $p_{2}$ have a non trivial intersection $T_{12}$, if and only if $1-1 / b_{1}-1 / b_{2}>0$, which is equivalent to the fact that the vertex $(1,1,0, \cdots, 0)$ of $K^{m}$ lies below the affine hyperplane determined by the face of $T$ opposite to $O$. The volume $V\left(T_{12}\right)$ of $T_{12}$ is equal to $\left(b_{1} b_{2} \cdots b_{m} / m !\right)\left(1-1 / b_{1}-\right.$ $\left.1 / b_{2}\right)^{m}$. Let $\left\{T_{12}, T_{13}, T_{23}, \cdots, T_{j h}\right\}$ be the set of all non trivial intersections of two outer simplexes. If three outer simplexes at $p_{1}, p_{2}$, and $p_{3}$ do not have non trivial intersecton $T_{123}$ (or equivalently, $\left.1-1 / b_{1}-1 / b_{2}-1 / b_{3} \leq 0\right)$, then $V\left(K^{m} \cap T\right)$ is given by $V(T)-V\left(T_{1}\right)-V\left(T_{2}\right)-\cdots-V\left(T_{s}\right)+V\left(T_{12}\right)+\cdots+V\left(T_{j h}\right)$.

(iii-3) If $T_{123}$ is non trivial, then we need the term $-V\left(T_{123}\right)=-\left(b_{1} b_{2} \cdots b_{m}\right.$ $/ m !)\left(1-1 / b_{1}-1 / b_{2}-1 / b_{3}\right)^{m}$ in the expression of $V\left(K^{m} \cap T\right)$.

(iii-4) Generally, the intersection $T_{t_{1} t_{2} \cdots t_{u}}$ of $T_{t_{1}}, T_{t_{2}}, \cdots, T_{t_{u}}$ is non trivial, if and only if $1-1 / b_{t_{1}}-1 / b_{t_{2}}-\cdots-1 / b_{t_{u}}>0$, and its volume has sign $(-1)^{u}$ in the expression of $V\left(K^{m} \cap T\right)$.

q.e.d.

Let $B^{m}$ be the unit cube in $E^{m}$ centered at the origin: $B^{m}=\left\{x ;-1 / 2 \leq x^{\}}\right.$ $\leq 1 / 2,1 \leq j \leq m\}$. Let $a=\left(a_{1}, a_{2}, \cdots, a_{m}\right)$ be a unit vector in $E^{m}$ and $H(a)$ be the hyperplane passing through the origin and orthogonal to $a$. Since the case where $a_{1}=0$ reduces to the lower dimensional case, we assume that the components of $a$ satisfy

$$
0<a_{1} \leq a_{2} \leq \cdots \leq a_{m-1} \leq a_{m} .
$$

Concerning the volume $V_{m}(a)$ of the slice $B^{m} \cap H(a)$ corresponding to $a$, Hensley [5] and Ball [1] gave the best possible inequality; $1 \leq V_{m}(a) \leq \sqrt{2}$, which was verified by using the following expression of $V_{m}(a)$ :

$$
V_{m}(a)=\frac{1}{\pi} \int_{-\infty}^{\infty} \prod_{k=1}^{m} \frac{\sin a_{k} t}{a_{k} t} d t
$$

Since (1.1) is homothetically invariant with respect to $\left(a_{\imath}\right)$, to prove Theorem A it suffices to give the value of the left hand side of (2.1). Namely we prove the following.

Proposition B. The volume $V_{m}(a)$ of the slice corresponding to a is given by

$$
V_{m}(a)=\frac{1}{a_{m}}-\frac{1}{c} \sum_{r=1}^{m-2}(-1)^{r-1} \sum_{r-t y p e} P_{r}(\lambda)^{m-1}
$$


where $c=(m-1) ! 2^{m-2} a_{1} a_{2} \cdots a_{m}$ and $P_{r}(\lambda)$ denotes a polynomial of $r$-type.

Proof. We define $\theta$ by $\cos \theta=\left\langle e_{m}, a\right\rangle=a_{m}$. By $\rho$ we denote the orthogonal projection of $E^{m}$ onto $E^{m-1}$ defined by $x_{m}=0$. First we study the case where $a_{m}>a_{1}+a_{2}+\cdots+a_{m-1}$. The condition $a_{m}>a_{1}+a_{2}+\cdots+a_{m-1}$ is equivalent to the fact that $H(a)$ does not meet the upper face $F^{m-1}$ of $B^{m}$ defined by $x_{m}=1 / 2$. Therefore, $\rho\left(B^{m} \cap H(a)\right)=B^{m-1}$. Hence, $V_{m}(a)=1 / \cos \theta=1 / a_{m}$.

Next we assume that $a_{m}<a_{1}+a_{2}+\cdots+a_{m-1}$ holds. Then $H(a)$ meets the upper face $F^{m-1}$. We denote the part of $F^{m-1}$ which lies below $H(a)$ by $K(a)$. Then the volume $V(K(a))$ of $K(a)$ is given by the preceding Lemma. The relation between $\left(b_{k}\right)$ and $\left(a_{k}\right)$ is given by $b_{k}=\left(a_{1}+a_{2}+\cdots+a_{m-1}-a_{m}\right) / 2 a_{k}, 1 \leq k \leq$ $m-1$. Consequently, we obtain the following:

$$
\begin{aligned}
& 1-1 / b_{k}=\left(a_{1}+a_{2}+\cdots \check{a}_{k} \cdots+a_{m-1}-a_{k}-a_{m}\right) / A, \\
& 1-1 / b_{k}-1 / b_{l}=\left(a_{1}+a_{2}+\cdots \check{a}_{k} \cdots \check{a}_{l} \cdots+a_{m-1}-a_{k}-a_{l}-a_{m}\right) / A,
\end{aligned}
$$

etc., where $A=a_{1}+a_{2}+\cdots+a_{m-1}-a_{m}$ and $\check{a}_{k}$ means that $a_{k}$ is removed. Since $V_{m}(a)=[1-2 V(K(a))] / \cos \theta$, we obtain (2.2). $\quad$ q. e. d.

\section{Corollaries}

First we give three special cases of Theorem A.

COROLlaRY C. For a positive number $a$ and integer $m \geq 2$, the following holds.

$$
\begin{aligned}
\int_{0}^{\infty} \frac{\sin ^{m} a t}{t^{m}} d t= & \frac{a^{m-1} \pi}{(m-1) ! 2^{m-1}}\left[(m-1) ! 2^{m-2}\right. \\
& \left.-\sum_{r=1}^{[(m-1) / 2]}(-1)^{r-1}{ }_{m-1} C_{r-1}(m-2 r)^{m-1}\right] .
\end{aligned}
$$

COROLlaRY D. For positive numbers $a<b$ and positive integers $u, v(u+v=$ $m)$, the following holds.

$$
\begin{aligned}
\int_{0}^{\infty} \frac{\sin ^{u} a t \sin ^{v} b t}{t^{m}} d t & =\frac{\pi}{(m-1) ! 2^{m-1}}\left[(m-1) ! 2^{m-2} a^{u} b^{v-1}\right. \\
- & \left.\sum_{r=1}^{m-2}(-1)^{r-1} \sum_{p=0}^{*}{ }_{u} C_{p} \cdot{ }_{v-1} C_{r-p-1}\{(u-2 p) a+(v-2 r+2 p) b\}^{m-1}\right],
\end{aligned}
$$

where $\Sigma^{*}$ denotes the sum over all polynomials $(u-2 p) a+(v-2 r+2 p) b>0$ and $p$ runs from $\max \{r-v, 0\}$ to $\min \{r-1, u\}$.

COROllary E. For positive numbers $a<b<c$ and positive integers $u, v, w(u+$ $v+w=m)$, the following holds: 


$$
\begin{aligned}
\int_{0}^{\infty} \frac{\sin ^{u} a t \sin ^{v} b t \sin ^{w} c t}{t^{m}} & d t=\frac{\pi}{(m-1) ! 2^{m-1}}\left[(m-1) ! 2^{m-2} a^{u} b^{v} c^{w-1}\right. \\
& \left.-\sum_{r=1}^{m-2}(-1)^{r-1} \sum_{p+q+s=r-1}^{*}{ }_{u} C_{p} \cdot{ }_{v} C_{q} \cdot{ }_{w-1} C_{s} \cdot P(p, q, s)^{m-1}\right],
\end{aligned}
$$

where $P(p, q, s)=(u-2 p) a+(v-2 q) b+(w-2 s-2) c$, and $\Sigma^{*}$ denotes the sum over all polynomials $P(p, q, s)>0$ and $0 \leq p \leq \min \{r-1, u\}, 0 \leq q \leq \min \{r-1, v\}$ and $0 \leq s=$ $r-1-p-q \leq w-1$.

Proof of Corollary $C$. The number of polynomials $a+a+\cdots+a-a$ of 1 -type is one for $m \geq 3$. The number of polynomials $a+a+\cdots+a-a-a$ of 2 -type is $m-1$ for $m \geq 5$. Similarly, the number of polynomials $a+a+\cdots+a-a-\cdots-a-a$ of $r$-type is ${ }_{m-1} C_{r-1}$. The range of $r$ is from 1 to $[(m-1) / 2]$. Therefore, Corollary $\mathrm{C}$ follows from Theorem A.

Proof of Corollary D. The number of polynomials of 1-type is at most one; $a+a+\cdots+a+b+\cdots+b-b$ for $m \geq 3$. Each of polynomials of 2-type is one of the following;

$$
\begin{array}{ll}
a+a+\cdots+a+a+b+\cdots+b-b-b & (u a+(v-4) b>0), \\
a+a+\cdots+a+b+\cdots+b+b-a-b & ((u-2) a+(v-2) b>0) .
\end{array}
$$

The numbers of such polynomials are ${ }_{u} C_{0} \cdot{ }_{v-1} C_{1}$ and ${ }_{u} C_{1} \cdot{ }_{v-1} C_{0}$. By $p$ we denote the number of $a$ with negative sign in the polynomial of $r$-type. Polynomials of $r$-type for general $r$ and the number of such polynomials are similarly studied.

Proof of Corollary $E$ is similar.

Corollary $\mathrm{C}$ enables us to calculate $I(m)$ for any $m$, for example, we obtain

$$
\begin{array}{ll}
I(7)=5887 a^{6} \pi / 23040, & I(8)=151 a^{7} \pi / 630, \\
I(9)=259723 a^{8} \pi / 1146880, & I(10)=15619 a^{9} \pi / 72576 .
\end{array}
$$

Also Corollary $\mathrm{D}$ enables us to calculate $I(u ; v)$ for any $u, v$, for example, we obtain

$$
\begin{aligned}
I(3 ; 4)= & \frac{\pi}{6 ! 2^{6}}\left[6 ! 2^{5} a^{3} b^{3}-(3 a+2 b)^{6}+3(3 a)^{6}+3(a+2 b)^{6}\right. \\
& \left.-3(3 a-2 b)^{6}-9 a^{6}-3(-a+2 b)^{6}\right] \quad(2 b \leq 3 a<3 b) \\
= & \frac{\pi}{6 ! 2^{6}}\left[6 ! 2^{5} a^{3} b^{3}-(3 a+2 b)^{6}+3(3 a)^{6}+3(a+2 b)^{6}\right. \\
& \left.-9 a^{6}-3(-a+2 b)^{6}+(-3 a+2 b)^{6}\right] \quad(3 a<2 b) .
\end{aligned}
$$

The formulas in Corollaries produce many analogous formulas. Here we give some examples. For convenience sake we use the following notations: 


$$
\begin{gathered}
I_{m}(u ; a)=\int_{0}^{\infty} \frac{\sin ^{u} a t}{t^{m}} d t, \\
I_{m}(u, v ; a, b)=\int_{0}^{\infty} \frac{\sin ^{u} a t \sin ^{v} b t}{t^{m}} d t, \\
I_{m}(u, v, w ; a, b, c)=\int_{0}^{\infty} \frac{\sin ^{u} a t \sin ^{v} b t \sin ^{w} c t}{t^{m}} d t .
\end{gathered}
$$

Then, for $m \geq 2$, we obtain the following:

COROLlaRY F.

$$
\begin{aligned}
\int_{0}^{\infty} \frac{\sin ^{m+2} a t}{t^{m}} d t=I_{m}(m ; a)-\frac{1}{4} I_{m}(m-2,2 ; a, 2 a), \\
\begin{aligned}
\int_{0}^{\infty} \frac{\sin ^{m} a t \sin ^{2} b t}{t^{m}} d t= & \frac{1}{2} I_{m}(m ; a)-\frac{1}{2} I_{m}(m-2,2 ; a, a+b) \\
& +\frac{1}{2} I_{m}(m-2,2 ; a, b)+\frac{1}{4} I_{m}(m-2,1,1 ; a, 2 a, 2 b),
\end{aligned}
\end{aligned}
$$

$$
\begin{aligned}
& \int_{0}^{\infty} \frac{\sin ^{m+4} a t}{t^{m}} d t=I_{m}(m+2 ; a)-\frac{1}{4} I_{m}(m, 2 ; a, 2 a), \\
& \int_{0}^{\infty} \frac{\sin ^{m} a t \cos ^{2} b t}{t^{m}} d t=I_{m}(m ; a)-I_{m}(m, 2 ; a, b), \\
& \int_{0}^{\infty} \frac{\sin ^{m} a t \cos b t}{t^{m}} d t=I_{m}(m-1,1 ; a, a+b)-\frac{1}{2} I_{m}(m-2,1,1 ; a, 2 a, b),
\end{aligned}
$$

etc.

In the above, (3.5) is somewhat complicated. If $m \geq 4$, then it has a simpler expression: $I_{m}(m-2, ; a, b)-(1 / 4) I_{m}(m-4,2,2 ; a, 2 a, b)$.

\section{REFERENCES}

[1] K. Ball, Cube slicing in $R^{n}$, Proc. Amer. Math. Soc., 97 (1986), 465-473.

[2] K. BALL, Some remarks on the geometry of convex sets, Lect. Notes in Math. (Springer) No. 1317 (1986-1987), 224-231.

[3] A. ERdÉlyi (Ed.), Tables of integral transforms, McGraw-Hill Book Comp. Inc. 1954.

[4] I. S. Gradshteyn and I. M. Ryzhik, Table of Integrals, Series, and Products. Academic Press, 1980.

[5] D. Hensley, Slicing the cube in $R^{n}$ and probability (Bounds for the measure of a central cube slice in $R^{n}$ by probability methods), Proc. Amer. Math. Soc., 73 (1979), 95-100.

[6] D. Hensley, Slicing convex bodies (Bounds for slice area in terms of the bodies' covariances), Proc. Amer. Math. Soc., 79 (1980), 619-625. 